\title{
862.
}

\section{NOTE ON THE THEORY OF LINEAR DIFFERENTIAL EQUATIONS.}

[From Crelle's Journal der Mathem., t. c. (1887), pp. 286-295.]

1. I CONSIDER a linear differential equation

$$
p_{0} \frac{d^{m} y}{d x^{m}}+p_{1} \frac{d^{m-1} y}{d x^{m-1}}+\ldots+p_{m} y=0
$$

where $p_{0}, p_{1}, \ldots, p_{m}$ are rational and integral functions of $x$, having no common factor: as usual, $x$ is a complex magnitude represented by a point, and we consider the integrals belonging to a singular point $x=a$ of the differential equation. An integral may be a regular integral, or it may be what Thomé calls a normal elementary integral: the theory of these integrals (which I would rather call subregular integrals) requires, I think, further examination.

2. I retain $x$ as the independent variable, but for shortness use $t$ to denote $\frac{1}{x-a}$ : and I take as the dependent variable $z,=\frac{1}{y} \frac{d y}{d x}$ : we have then $z$ determined by a non-linear differential equation of the order $m-1$, and from any value of $z$ we derive a corresponding value $e^{\int z d x}$ of $y$.

3. To obtain the $z$-equation, using for shortness accents to denote differentiation in regard to $x$, we have $z=\frac{y^{\prime}}{y}$, and thence $z^{\prime}=\frac{y^{\prime \prime}}{y}-\left(\frac{y^{\prime}}{y}\right)^{2}$, that is, $z^{2}+z^{\prime}=\frac{y^{\prime \prime}}{y}$ : hence also $2 z z^{\prime}+z^{\prime \prime}=\frac{y^{\prime \prime \prime}}{y}-\frac{y^{\prime} y^{\prime \prime}}{y^{2}}$, that is,

$$
z\left(z^{2}+z^{\prime}\right)+2 z z^{\prime}+z^{\prime \prime}=\frac{y^{\prime \prime \prime}}{y}, \text { or finally } z^{3}+3 z z^{\prime}+z^{\prime \prime}=\frac{y^{\prime \prime \prime}}{y}
$$

and so on; viz. the values of $\frac{y^{\prime}}{y}, \frac{y^{\prime \prime}}{y}, \frac{y^{\prime \prime \prime}}{y}, \ldots$ are $z, z^{2}+z^{\prime}, z^{3}+3 z z^{\prime}+z^{\prime \prime}, \ldots$; generally for 
$\frac{y^{(n)}}{y}$ the first term is $z^{n}$ and the last term is $z^{(n-1)}$. If, to fix the ideas, the $y$-equation is

$$
p_{0} y^{\prime \prime \prime}+p_{1} y^{\prime \prime}+p_{2} y^{\prime}+p_{3}=0,
$$

then, dividing by $y$, the $z$-equation is

$$
p_{0}\left(z^{3}+3 z z^{\prime}+z^{\prime \prime}\right)+p_{1}\left(z^{2}+z^{\prime}\right)+p_{2} z+p_{3}=0
$$

and similarly, from the $y$-equation of the order $m$, we derive a $z$-equation of the order $m-1$,

$$
p_{0}\left\{z^{m}+\ldots+z^{(m-1)}\right\}+\ldots+p_{m-2}\left(z^{2}+z^{\prime}\right)+p_{m-1} z+p_{m}=0 .
$$

4. In this equation, each coefficient is in the first instance a rational and integral function of $x$, or, what is the same thing, of $x-a$; writing $t$ to denote $\frac{1}{x-a}$, each coefficient is thus the sum of a finite number of negative integer powers of $t$; hence multiplying the whole equation by a proper positive power of $t$, the several coefficients become each of them the sum of a finite number of positive powers of $t$, or arranging in descending powers of $t$, say the general form is $p_{r}=\alpha_{r} t^{\lambda}+\beta_{r} t^{\lambda-1}+\ldots+\kappa_{r}$, where $\lambda$ is a positive integer which may be $=0$, and which has for each coefficient its proper value. We wish to satisfy the $z$-equation by a value $z=$ descending series in $t$, viz. the form is $z=A t^{a}+A^{\prime} t^{a-a^{\prime}}+\ldots$, with powers which are integral or fractional, but where the number of positive powers is finite. The theory is almost identical with that of the solution in like form of the algebraical equation

$$
p_{0} z^{m}+p_{1} z^{m-1}+\ldots+p_{m-1} z+p_{m}=0
$$

or say the equation $U=0$, where $U$ is a rational and integral function of $z$ and $t$, of the degree $m$ as regards $z$.

5. Considering $z$ and $t$ as Cartesian coordinates, the equation in question, $U=0$, represents a geometrical curve such for any given value of the abscissa $t$, the ordinate $z$ has $m$ values: the curve is of the order $m$ at least, but it may be of a higher order $m+\kappa$; when this is so, there is at infinity on the axis $t=0$, a $\kappa$-tuple point $K$, and thus any line parallel to the axis $t=0$, intersects the curve in the point $K$ counting as $\kappa$ intersections, and in $m$ other points, which are the points belonging to the $m$ values of the ordinate $z$. Taking $s=1$, and for $z, t$ writing $\frac{z}{s}, \frac{t}{s}$ respectively, we have between the trilinear coordinates $z, t, s$ an equation $U^{\prime}=0$ of the order $m+\kappa$, where $s=0$ is the equation of the line infinity: and the curve has a $\kappa$-tuple point at the point $K$ for which $t=0, s=0$.

6. Starting from the equation $U=0$, for an arbitrary value of $t$ we have $m$ values of $z$ all of them different. These may be developed, each of them as a descending series in $t$, and the development is effected in the usual manner; viz. considering for the moment $t$ as very large, then $z$ is in general very large and it has a leading term $A t^{a}$, which is determined by writing in the equation $z=A t^{a}$, and then finding $\alpha$ in such wise that there are two or more exponents equal to each other and greater 
(that is, nearer $+\infty$ ) than any other exponent: we have thus a highest power of $t$, the whole coefficient for which must vanish, viz. we obtain an equation of two or more terms giving for $A$ a value or values not $=0$; in the term $A t^{a}$ in question, $\alpha$ is in general positive, but it may be $=0$, or be negative. The leading term $A t^{a}$ being found in this manner, the law for the exponents of the subsequent terms is frequently at once apparent; but, if necessary, we write $z=A t^{a}+A^{\prime} t^{a-a^{\prime}}$, and determine in like manner the exponent $\alpha-\alpha^{\prime}$ and the coefficient $A^{\prime}$. Proceeding in this manner until the law of the exponent becomes apparent, and then by the method of indeterminate coefficients, we finally arrive at a series

$$
z=A t^{\alpha}+A^{\prime} t^{\alpha-\alpha^{\prime}}+A^{\prime \prime} t^{\alpha-\alpha^{\prime}-\alpha^{\prime \prime}}+\ldots,
$$

in descending powers of $t$ : the exponents are integral or fractional, but the number of positive exponents is always finite. There is either a single series or it may be two or more series; but the number of series is at most $=m$, and when it is less than $m$, then the coefficients in the several series or some of them will contain radicals, and by giving to each radical its different values, the system of series will determine $m$ different values of $z$.

7. The curve meets the line infinity $(s=0)$ in the point $K$ counting as $\kappa$ intersections, and in $m$ other points, some or all of which may coincide with $K$; the forms of the series depend on the configuration of the $m$ points, or say on the relation of the curve to the line infinity. Thus suppose $\kappa=0$, and further that the $m$ points are all of them distinct, that is, let the curve be a curve of the order $m$ meeting the line infinity in $m$ distinct points, or, what is the same thing, having $m$ asymptotes, no two of them parallel; we have in this case $m$ series, each of the form

$$
z=A t+B+\frac{C}{t}+\frac{D}{t^{2}}+\ldots
$$

where the several coefficients $A$ have distinct values.

8. In the foregoing case $A$ is determined by an equation of the order $m$, having unequal roots; and taking for $A$ any root at pleasure, the remaining coefficients $B, C, \ldots$ are each of them linearly determined. If the equation has two equal roots, this may correspond to the case of two parallel asymptotes (the curve has here a node at infinity); the coefficients $B, C, \ldots$ will in this case depend on a quadric radical, and by giving to this radical its two values, we obtain the two series

$$
\begin{aligned}
& z=A t+B+\frac{C}{t}+\ldots \\
& z=A t+B^{\prime}+\frac{C^{\prime}}{t}+\ldots
\end{aligned}
$$

corresponding to the two equal roots of the equation. But if instead of a node at infinity we have the line infinity a tangent to the curve, then instead of the two parallel asymptotes, we have an asymptotic parabola; the series assumes a new form, viz. it contains terms in $t^{\frac{1}{3}}, t^{-\frac{1}{2}}, \ldots$; the coefficients of the integer powers have 
determinate values, but those of the powers $t^{\frac{1}{2}}, t^{-\frac{1}{2}}, \ldots$ contain as factor a quadric radical, and giving to this radical its two values, we have two series

$$
\begin{aligned}
& z=A t+C+\ldots+t^{\frac{1}{2}}\left(B+\frac{D}{t}+\ldots\right) \\
& z=A t+C+\ldots-t^{\frac{1}{2}}\left(B+\frac{D}{t}+\ldots\right)
\end{aligned}
$$

9. The like considerations apply in the case where the line infinity passes through higher multiple points of the curve, or has with it a contact or contacts other than a single ordinary contact: in every case, reckoning as distinct series those obtained by attaching to each radical its different values, the number of distinct series will be $=m$ precisely.

10. Reverting now to the differential equation

$$
p_{0}\left\{z^{m}+\ldots+z^{(m-1)}\right\}+\ldots+p_{m-2}\left(z^{2}+z^{\prime}\right)+p_{m-1} z+p_{m}=0,
$$

it is to be observed that the very same process is applicable to the determination of $z$ in the form of a descending series $z=A t^{a}+A^{\prime} t^{a-a^{\prime}}+\ldots$; moreover, it frequently happens that the determination of the leading term $A t^{\alpha}$ is made by means of the algebraical equation

$$
p_{0} z^{m}+\ldots+p_{m-2} z^{2}+p_{m-1} z+p_{m}=0
$$

viz. this is so whenever the value of $\alpha$ is greater than 1 : in fact, writing $z=A t^{a}(\alpha>1)$, then in the sets of terms $\left\{z^{m}+\ldots+z^{(m-1)}\right\}, \ldots,\left(z^{3}+3 z z^{\prime}+z^{\prime \prime}\right),\left(z^{2}+z^{\prime}\right)$, the first terms $z^{m}, \ldots, z^{3}, z^{2}$ are each of them of a higher degree than any other term in the same brackets, so that attending only to the terms of highest degree the sets may be reduced to $z^{m}, \ldots, z^{3}, z^{2}$. But in the determination of the subsequent exponents and coefficients, the omitted terms or some of them would of course come into play: and it is at least not obvious that we can, for the forms of the series which satisfy the differential equation, employ geometrical considerations such as those which were used in regard to the series satisfying the algebraical equation.

11. Considering as above the coefficients $p_{0}, p_{1}, \ldots, p_{m}$ as rational and integral functions of $t$, it is easy to determine the degrees of these coefficients in such wise that the exponent $\alpha$ of the leading term $A t^{a}$ shall have a given value. Suppose first, this given value is $\alpha=a$, a positive integer greater than 1 : the degrees may be $\theta, \theta+a, \theta+2 a, \ldots, \theta+m a,(\theta=0$ or any positive integer at pleasure); and not only so, but if we write

$$
p_{0}=L_{0} t^{\theta}+\ldots, \quad p_{1}=L_{1} t^{\theta+\mathrm{a}}+\ldots, \quad p_{m}=L_{m} t^{\theta+m a}+\ldots,
$$

then writing $z=A t^{\mathrm{a}}$, clearly the highest power in the equation is $t^{\theta+m a}$, and equating the coefficient hereof to zero, we have

$$
L_{0} A^{m}+L_{1} A^{m-1}+\ldots+L_{m-1} A+L_{m}=0
$$


an equation of the degree $m$ for the determination of the coefficient of the leading term $A t^{\mathrm{a}}(\mathrm{a}>1)$. Assuming that the roots are all unequal, we have $m$ series each of the form

$$
z=A t^{\mathrm{a}}+B t^{\mathrm{a}-1}+\ldots+K+\frac{L}{t}+\ldots
$$

12. Suppose $\mathrm{a}=1$, that is, let the leading term be $A t$; here the degrees may be $\theta, \theta+1, \theta+2, \ldots, \theta+m,(\theta=0$ or any positive integer as before), but we have a different form for the $A$-equation. In fact, here $(z=A t)$ in the sets of terms $\left\{z^{m}+\ldots+z^{(m-1)}\right\}, \ldots,\left(z^{3}+3 z z^{\prime}+z^{\prime \prime}\right),\left(z^{2}+z^{\prime}\right)$, the terms in each brackets are of the same degree, viz. the degrees are $m, \ldots, 3,2$; and not only so, but we have $z^{2}+z^{\prime}=\left(A^{2}+A\right) t^{2}$, that is, $=[A]^{2} t^{2}, z^{3}+3 z z^{\prime}+z^{\prime \prime}=[A]^{3} t^{3}, \ldots$. Hence if

the $A$-equation is

$$
p_{0}=L_{0} t^{\theta}+\ldots, \quad p_{1}=L_{1} t^{\theta+1}+\ldots, \quad p_{m}=L_{m} t^{\theta+m}+\ldots,
$$

$$
L_{0}[A]^{m}+L_{1}[A]^{m-1}+\ldots+L_{m-1}[A]^{1}+L_{m}=0,
$$

an equation of the degree $m$ as before; assuming that the roots are unequal, we have here $m$ series each of the form

$$
z=A t+B+\frac{C}{t}+\frac{D}{t^{2}}+\ldots
$$

being, as will presently appear, the case of $m$ regular integrals for the $y$-equation.

13. In either of the last-mentioned cases, the formula for the $A$-equation holds good if any two or more of the coefficients $p_{0}, p_{1}, \ldots, p_{m}$ are of the degrees aforesaid, the others of them being of inferior degrees; we have only to put $=0$ the $L$ 's which belong to the coefficients of inferior degrees. If neither $L_{0}$ nor $L_{m}$ be $=0$, the equation is still an equation of the order $m$, with $m$ effective roots; but if any of the coefficients $L$ at the beginning of the equation vanish we thus introduce roots $=\infty$ : and if any of the coefficients $L$ at the end of the equation vanish, we thus introduce roots $=0$ : the number of effective roots is $=m$-the number of roots $\infty$ or 0 ; the case requires further consideration. If the equation has equal roots, then from what precedes, it is easy to infer that we may have distinct series containing as in the general case only integer powers of $t$, or we may have series beginning with $A t^{\mathrm{a}}$ or $A t$, but containing also fractional powers of $t$.

14. If the leading term is $z=A t^{a}, \alpha=a$, a value $<1$, then in the sets of terms $\left\{z^{m}+\ldots+z^{(m-1)}\right\}, \ldots,\left(z^{3}+3 z z^{\prime}+z^{\prime \prime}\right),\left(z^{2}+z^{\prime}\right)$ the last terms $z^{(m-1)}, \ldots, z^{\prime \prime}, z^{\prime}$ are each of them of a higher degree than any other term in the same brackets, so that attending only to the terms of the highest degree the sets may be reduced to $z^{(m-1)}, \ldots, z^{\prime \prime}, z^{\prime}$, or we may consider the equation

$$
p_{0} z^{(m-1)}+p_{1} z^{(m-2)}+\ldots+p_{m-2} z^{\prime}+p_{m-1} z+p_{m}=0 .
$$

In particular, this is the case for $z=A t^{-\mathrm{a}}$, a being a positive integer; in this case, the degrees of $p_{0}, p_{1}, \ldots, p^{m-1}, p_{m}$ may be $\theta+\mathrm{a}-m+1, \theta+\mathrm{a}-m+2, \ldots, \theta+\mathrm{a}, \theta$; and this being so, the leading coefficient $A$ will be determined by a linear equation. But the case is, in fact, that of a non-singular point $x=a$ : and I do not here further consider it. 
15. Reverting to the case where the series is

$$
z=A t+B+\frac{C}{t}+\frac{D}{t^{2}}+\ldots
$$

and substituting for $t$ its value $\frac{1}{x-a}$, or for greater convenience $\frac{1}{x}$, this is

whence

$$
\frac{1}{y} \frac{d y}{d x}=\frac{A}{x}+B+C x+D x^{2}+\ldots
$$

$$
\log y=A \log x+B x+\frac{1}{2} C x^{2}+\ldots,
$$

or passing to the value of $y$, but expanding the exponential of $B x+C x^{2}+\ldots$, this is

$$
y=x^{A}\left(1+B^{\prime} x+C^{\prime} x^{2}+\ldots\right),
$$

viz. we have here a regular integral. By what precedes, it appears that, for the values $p_{0}=L_{0} x^{-\theta}+\ldots, p_{1}=L_{1} x^{-\theta-1}+\ldots, p_{m}=L_{m} x^{-\theta-m}+\ldots$, the exponent $A$ is determined by the equation

$$
L_{0}[A]^{m}+L_{1}[A]^{m-1}+\ldots+L_{m-1}[A]^{1}+L_{m}=0,
$$

which is the equation for that purpose considered by Fuchs, and is what I would call the indicial equation.

The logarithmic forms which belong to the case of equal roots may be deduced from the case of unequal roots, by supposing two or more of the roots to approach each other continuously and become ultimately equal.

16. Similarly, if the series be

$$
z=F t^{\mathrm{a}}+\ldots+K t^{2}+A t+B+\frac{C}{t}+\ldots
$$

(a being a positive integer $=2$ at least), then writing as above $t=\frac{1}{x}$, this is

$$
\frac{1}{y} \frac{d y}{d x}=\frac{F}{x^{2}}+\ldots+\frac{K}{x^{2}}+\frac{A}{x}+B+C x+\ldots
$$

whence, if for shortness $w=-\frac{F}{a-1} \frac{1}{x^{\mathrm{a}-1}}-\ldots-\frac{K}{x}$, we have

$$
\log y=w+A \log x+B x+\frac{1}{2} C x^{2}+\ldots,
$$

or passing to the value of $y$, but expanding as before the exponential of $B x+\frac{1}{2} C x^{2}+\ldots$, this is

$$
y=e^{w} x^{A}\left(1+B^{\prime} x+C^{\prime} x^{2}+\ldots\right)
$$

a subregular integral. By what precedes, it appears that, if

C. XII

$$
p_{0}=L_{0} x^{-\theta}+\ldots, p_{1}=L_{1} x^{-\theta-\mathrm{a}}+\ldots, p_{m}=L_{m} x^{-\theta-m \mathrm{a}}+\ldots,
$$


then the equation for the leading coefficient $F$ (previously represented by $A$ ) is

$$
L_{0} F^{m}+L_{1} F^{m-1}+\ldots+L_{m-1} F+L_{m}=0
$$

but I do not see any direct or simple way of obtaining the corresponding equation for the exponent $A$ of this subregular integral.

17. To illustrate the case of a series with fractional powers, and also in order to work out a simple example with some completeness, I consider the differential equation of the second order $\frac{d^{2} y}{d x^{2}}+p_{2} y=0$, giving the z-equation $z^{2}+z^{\prime}+p_{2}=0 ; p_{2}$ is a rational and integral function of $t\left(=\frac{1}{x}\right)$, and we have two cases according as the order of the function is even or odd.

18. Suppose first that the order is even, and for convenience taking it to be $=4$, and assuming the value of $p_{2}$ accordingly, I consider the equation

$$
z^{2}+z^{\prime}=\alpha t^{4}+\beta t^{3}+\gamma t^{2}+\delta t+\epsilon .
$$

Here the form is at once seen to be

and observing that we have

$$
z=A t^{2}+B t+C+\frac{D}{t}+\frac{E}{t^{2}}+\ldots
$$

we find

$$
z^{\prime}=-t^{2} \frac{d z}{d t}=-2 A t^{3}-B t^{2}+D+\frac{2 E}{t}+\ldots
$$

equations which give

$$
\begin{aligned}
& A^{2}=\alpha, \\
& 2 A B-2 A=\beta \text {, } \\
& 2 A C+B^{2} \quad-B=\gamma, \\
& 2 A D+2 B C+0 C=\delta \text {, } \\
& 2 A E+2 B D+C^{2}+D=\epsilon \text {, } \\
& 2 A F+2 B E+2 C D+2 E=0 \text {, }
\end{aligned}
$$

$$
\begin{aligned}
A= & \sqrt{\alpha} \\
B= & 1+\frac{\beta}{2 \sqrt{\alpha}}, \\
C= & \frac{\gamma}{2 \sqrt{\alpha}}-\frac{\beta}{4 \alpha}-\frac{\beta^{2}}{8 \alpha \sqrt{\alpha}}, \\
D= & \frac{\delta}{2 \sqrt{\alpha}}-\frac{\gamma}{2 \alpha}+\frac{1}{4 x \sqrt{\alpha}}(\beta-\beta \gamma)+\frac{\beta^{2}}{4 \alpha^{2}}+\frac{\beta^{3}}{16 \alpha^{2} \sqrt{\alpha}}, \\
E= & \frac{\epsilon}{2 \sqrt{\alpha}}-\frac{3 \delta}{4 \alpha}+\frac{1}{4 \alpha \sqrt{\alpha}}\left(3 \gamma-\beta \delta-\frac{1}{2} \gamma^{2}\right)+\frac{1}{4 \alpha^{2}}\left(-\frac{3}{2} \beta+3 \beta \gamma\right) \\
& +\frac{1}{16 \alpha^{2} \sqrt{\alpha}}\left(-\frac{17}{2} \beta^{2}+3 \beta^{2} \gamma\right)-\frac{\beta^{3}}{4 \alpha^{3}}-\frac{5}{128} \frac{\beta^{4}}{\alpha^{3} \sqrt{\alpha}} ; \& c .
\end{aligned}
$$


We then have

and consequently

$$
\frac{1}{y} \frac{d y}{d x}=\frac{A}{x^{2}}+\frac{B}{x}+C+D x+E x^{2}+\ldots
$$

$$
y=e^{-\frac{A}{x}} x^{B} e^{C x+\frac{1}{2} D x^{2}+\frac{1}{3} E x^{3}+\ldots},
$$

where the second exponential is to be expanded in the form $1+C^{\prime \prime} x+D^{\prime} x^{2}+\ldots$; there are of course two values, say $y_{1}$ and $y_{2}$, corresponding to the values $+\sqrt{\alpha}$ and $-\sqrt{\alpha}$ of the radical respectively.

19. Secondly, if the order be odd, and for convenience taking it to be $=3$, and assuming the value of $p_{2}$ accordingly, I consider the equation

$$
z^{2}+z^{\prime}=\beta t^{3}+\gamma t^{2}+\delta t+\epsilon .
$$

Here the series is at once seen to be

we have

$$
z=A t^{\frac{3}{2}}+B t+C t^{\frac{1}{2}}+D+E t^{-\frac{1}{2}}+\ldots
$$

$$
z^{\prime}=-\frac{3}{2} A t^{\frac{5}{2}}-B t^{2}-\frac{1}{2} C t^{\frac{3}{2}}+\frac{1}{2} E t^{\frac{1}{2}}+\cdots,
$$

and we thence derive the equations

$$
\begin{aligned}
& A^{2} \quad=\beta \text {, } \\
& 2 A B-\quad-\frac{3}{2} A=0 \text {, } \\
& 2 A C+B^{2}-B=\gamma, \\
& 2 A D+2 B C \quad-\frac{1}{2} C=0, \\
& 2 A E+2 B D+C^{2}=\delta, \\
& 2 A F+2 B E+2 C D+\frac{1}{2} E=0,
\end{aligned}
$$

(where in the next equation we have on the right-hand side $\epsilon$, and in each of the subsequent equations we have on the right-hand side 0 ). We find

$$
\begin{aligned}
& A=\sqrt{\beta}, \\
& B=\frac{3}{4}, \\
& C=\frac{1}{2 \sqrt{\beta}}\left(\gamma+\frac{3}{16}\right), \\
& D=-\frac{1}{4 \beta}\left(\gamma+\frac{3}{16}\right), \\
& E=\frac{1}{2 \beta \sqrt{\beta}}\left(\delta+\frac{9}{32} \gamma-\frac{1}{4} \gamma^{2}+\frac{63}{1024}\right), \quad \& c .,
\end{aligned}
$$

where observe that the coefficients $A, C, E, \ldots$ contain as a factor $\frac{1}{\sqrt{\beta}}$, but the coefficients $B, D, \ldots$ are rational. 
We have

$$
z=\frac{1}{y} \frac{d y}{d x}=\frac{A}{x^{\frac{3}{2}}}+\frac{B}{x} \quad+\frac{C}{x^{\frac{1}{2}}}+D+E x^{\frac{1}{2}}+F x+\ldots
$$

giving

$$
\log y=-\frac{2 A}{x^{\frac{1}{2}}}+B \log x+2 C x^{\frac{1}{2}}+D x+\frac{2}{3} E x^{\frac{3}{2}}+\frac{1}{2} F x^{2}+\ldots
$$

and thence passing to the value of $y$, but expanding the exponential of $2 C x^{\frac{1}{2}}+D x+\ldots$, we have say the integral

$$
y_{1}=e^{-\frac{2 A}{x^{\frac{1}{2}}}} x^{B}\left(1+C^{\prime} x^{\frac{1}{2}}+D^{\prime} x+E^{\prime} x^{\frac{3}{2}}+\ldots\right)
$$

and then also, changing the sign of $\sqrt{\beta}$, the integral

$$
y_{2}=e^{\frac{2 A}{x^{\frac{1}{2}}}} x^{B}\left(1-C^{\prime} x^{\frac{1}{2}}+D^{\prime} x-E^{\prime} x^{\frac{3}{2}}+\ldots\right) .
$$

Writing for a moment

$$
\begin{aligned}
& Q=e^{-\frac{2 A}{x^{\frac{1}{2}}}} \\
& M=1+D^{\prime} x+F^{\prime} x^{2}+\ldots \\
& N=C^{\prime}+E^{\prime} x+G^{\prime} x^{2}+\ldots
\end{aligned}
$$

the two integrals are $x^{B} Q\left(M+N x^{\frac{1}{2}}\right)$, and $x^{B} Q^{-1}\left(M-N x^{\frac{1}{2}}\right)$ : these may be replaced by their sum and difference, viz. these are

$$
\begin{aligned}
& y_{1}+y_{2}=M x^{B}\left(Q+Q^{-1}\right)+N x^{B+\frac{1}{2}}\left(Q-Q^{-1}\right), \\
& y_{1}-y_{2}=M x^{B}\left(Q-Q^{-1}\right)+N x^{B+\frac{1}{2}}\left(Q+Q^{-1}\right),
\end{aligned}
$$

where $Q+Q^{-1}$ is a rational function of $x$, and $Q-Q^{-1}$ is $=x^{\frac{1}{2}}$ multiplied by a rational function of $x$; hence $y_{1}+y_{2}$ is $=x^{B}$ multiplied by a rational function of $x$, but $y_{1}-y_{2}$ is $=x^{B+\frac{1}{2}}$ multiplied by a rational function of $x$.

Cambridge, 28 April 1886. 